frame, say an eighth of an inch, separated the two plates from each other.

On taking the $\mathrm{n}$ out of the case the other day I noticed the pattern on the glass clearly and sharply imprinted on the ebonite ; every little circle well marked. Dust had been plentifully deposited on all parts not screened by the tinfoil spots, and the striking clearness of the impression was mainly due to this local absence of dust; but even on wiping off some of the dust the pattern could still be detected, owing to some difference of surface between the exposed and the shaded portions.

It evidently is another illustration of Prof. McLeod's observation of the effect of light on ebonite, the modified surface affording an easy lodgment for dust. In case there be anything more in the matter it is proposed to replace the same or similar plates, and observe at intervals.

EDWARD E. Robinson

Lecture Assistant to the Professor of Physics in University College, Liverpool

\section{THE SLIDE RULE}

I $\mathrm{T}$ is a perpetual source of amazement to those who are familiar with this instrument that its use is not almost universal. People of every class have to make simple calculations, while those engaged in scientific work, in designing apparatus, or in invention perpetually cover sheets of paper with figures, all of which trouble and the loss of time which it involves might be saved by the intelligent use of a good slide rule, and yet, for reasons difficult to find out, the habitual use of this instrument is limited to a very small proportion of the calculating community.

Most people know that the scales are logarithmically divided-that is, that the distance between the divisions marked I and ro being in imagination divided into Io, oo parts, the division marked 2 is at the 30 ooth of these parts, the division marked 3 is at the 477 Ist of these parts, and so on, 3010 being the $\log$. of 2, 477 I the log. of 3 , and so on ; and further, that the spaces between these whole numbers are similarly divided into fractional parts, thus $I \cdot I$ is at the 4I th of the imaginary parts and $\mathrm{I}^{\circ} \mathrm{O}$ a the 43rd of these parts, $4 \mathrm{I} 4$ and $\mathrm{I} 3$ being the logs. of $\mathrm{I}^{\prime} \mathrm{I}$ and $\boldsymbol{I}$ 'OI. This is very generally known, but it is more generally believed that to use the rule involves so much thought and anxiety that it is far simpler to work out results in the usual way, or at any rate that the rule can only be of any real assistance when a great number of similar calculations have to be made; and further that, as the results to be obtained are not absolutely correct, that as an extreme error of I, I-Ioth, or I-Iooth per cent. is possible, according to the nature of the instrument, it is not really to be trusted. These objections are easily answered. As soon as the slight difficulty of reading the rule has been overcome-a difficulty due to the fact that in ascending the scale the divisions become closer, so that if there is room for ten subdivisions between IO and II, there are only five between 20 and $2 \mathrm{I}$, and two between 40 and $4 \mathrm{I}-\mathrm{a}$ difficulty which once overcome never recursthen the simpler calculations, such as multiplication, division, and simple proportion, can at all times without an effort or a thought be instantly performed, while those involving proportions in which some of the terms are squares, cubes, roots, sines, or tangents can, after a moment's reflection, be as easily completed, so that even in the case of single operations time is saved. It is true when many calculations of the same kind present themselves, especially if some of the terms in the series are identical, that the use of the rule is specially advantageous; but in any case mental labour and time are saved.

As to the probable accuracy of results obtained by the use of the rule, they are in general superior to the accuracy with which the figures which require reduction have been determined, or, if this is not the case, they are in general so nearly correct that the error is of no con- sequence. For instance, if the marks obtained by several examinees are to be reduced to correspond to a total of Ioo, the commonest rule, which gives an accuracy of I-3ooth part, is sufficiently good; for the nearest whole number only, and the right order are all that are needed. It would be absurd to doubt the accuracy of the instrument because it cannot be trusted to give figures correct to one part in a thousand. Or, again, if the weight of a piece of metal has to be determined from its dimensions, a good rule trustworthy to I part in 1000 will in almost every case be more than good enough; for, even if the specific gravity of the material be known so truly, it is not often that the piece can be made so near the specified size that the discrepancy which may ultimately be observed will be due more to the error of the rule than to the inaccuracy of construction. In such a case it would be as absurd to discard the rule as untrustworthy as it is to use 7 -figure logarithms for the calculations of an ordinary chemical analysis. There are cases, of course, where observations can be made with a degree of accuracy beyond that which is obtainable by any rule-for instance, determinations of mass, length, angles, and time can all be made with extraordinary precision. Where, then, uncertainty is not introduced by observations of another kind, where the entire precision to be obtained in any such observations may be expected in the result, as, for instance, in the determination of the refractive index of the glass of a prism, in such cases the slide rule is unsuitable, and tables of logarithms furnish the most obvious means of making the calculations. Or, again, when pounds, shillings, and pence are involved, a result correct to the nearest farthing is generally desired to make accounts come right, and so, unless the sums dealt with are moderate, the slide rule is again unsuitable. However, the calculation of interest furnishes a good example of proper and improper use of the rule in making calculations. If it is required to find what a certain sum $(s)$ will be worth at the end of a year at so much $(r)$ per cent., the result might be found from the proportion $100: 100+r:: s: x$. Here the amount $x$ would be determined with an accuracy of say I-Ioooth part, so that if Ioool. were involved, an error of 17 . might arise. This is an improper use of the rule. A greater degree of accuracy would be obtained by the proportion $100: r:: s:$ the increase of $s$. Here the interest is found to the same proportionate accuracy, and so in such a case the greatest possible error could only be one shilling, if the rate is 5 per cent. This example, though obvious, is given because it corresponds exactly with cases that arise in the laboratory, where the rule, if used properly, is of service, but, if improperly, is useless.

Calculations involving only the simple arithmetical rules, when extreme accuracy is required, are best performed by the help of a table of logarithms, or with an arithmometer; in fact with an arithmometer a far greater degree of accuracy can be reached than with ordinary 7-figure logarithms, and though they are also suitable for calculations in which only three or four significant figures are required, their great size and expense compare unfavourably with the portability and cheapness of the rule, and, moreover, trigonometrical and logarithmic functions cannot be found with them. These machines are shown at the Inventions Exhibition by Tate and Edmonson, and are worth examining. There is another calculating machine close to Tate's, by which the interest on any sum at any rate per cent. for any time may be found to the nearest halfpenny in an incredibly short space of time, worthy of the attention of those who have to calculate interest. But, to return to the slide-rule, it is astonishing that an instrument like Gravet's, Io inches long only, with which all calculations, arithmetical, trigonometrical, and logarithmic, can be worked out so easily and with an accuracy of from I-500 to I-I000, according to the nature of the calculation, should be so little used. 
This is not the place to give instructions for using the rule, but an outline of the method is necessary to make it possible to compare the different makes, many of which are shown at the Inventions Exhibition.

With two similar scales of equal parts, as inches divided into tenths or centimetres divided into millimetres, it is possible to add numbers, or, conversely, to subtract numbers; thus, if the zero of one scale is placed opposite, say, 6.5 of the other, opposite every number $n$ on the first will be found $n+6.5$ on the second, and so addition or subtraction could be performed, but there would be no advantage in so adding or subtracting. In the same way the slide of the ordinary slide rule is employed to add distances, but these distances do not correspond to the figures attached, but to the logarithms of those figures, and so the sum which is found by such an addition is not the sum of the figures apparently added, but their product. If the slide is placed at random, all the pairs of figures which are opposite to one another are in the same proportion, and the multipliers which will change either series into the other will be found on each scale opposite the divisions marked I on the other. It requires no great amount of memory to bear this in mind: however the slide may be set, those numbers which are opposite to one another are in the same proportion, i.e. have a common quotient; which may be found opposite any of the divisions marked 1 ; and yet this is all that has to be remembered in multiplication, division, and simple proportion. The two top lines of a slide rule are generally identical, and they are used for these simple operations; they are generally distinguished by the letters $A$ and $B$. In general the bottom line of the slide, that is, the third altogether, is identical with the first two, and is labelled C. This arrangement is convenient, for it is possible to insert the slide upside down, in which case all numbers which are opposite one another on $\mathrm{A}$ and $\mathrm{C}$ have a common product, which may be found opposite any of the divisions marked I. This furnishes the most ready mode of finding actual or approximate factors of numbers, and is of great use to those who have to calculate wheelwork; further, by the use of the inverted $C$ line under the A line any harmonical progression can at once be read, and any number of harmonic means can be inserted between two quantities. The fourth line is generally made different from the others in that it is on double the scale, and it is then distinguished by the letter $D$. If the units of the $C$ and $D$ line are placed opposite one another, a table of squares and roots is formed, or if in any other position the squares of the numbers on $\mathrm{D}$ vary in the same proportion as do the numbers that are opposite to them on C. It is in calculations made on the $C$ and $D$ lines that so much time is saved, for proportions in which some of the terms are squares or square roots can be worked out as quickly and as accurately as those in which simple numbers only are employed. If the slide is inverted so as to bring the $\mathrm{B}$ line opposite to the $\mathrm{D}$ line, then the square of any number on $\mathrm{D} \times$ the number opposite to it on $\mathrm{B}$ is constant. This product may of course be found in $\mathrm{B}$ opposite $\mathrm{I}$ in $\mathrm{D}$. Cube roots, among other things, may be found in this way.

These four lines are all that are generally found in a slide rule; occasionally others are added: thus a line on one third of the scale of the D line (sometimes called an $\mathrm{E}$ line) will, with the $\mathrm{D}$ line, enable one to directly work proportions in which some of the terms are cubes or cube roots, but this is not often required. With the usual four lines all arithmetical processes, except addition and subtraction, can be performed. There are, however, rules in which on the back of the slide are scales in which the distances are log. sines or log. tangents of the angles marked, then these lines being placed against an ordinary A line so that $90^{\circ}$ on the line of sines or $45^{\circ}$ on the line of tangents is opposite I on the A line, a table of sines or tangents will be formed; and if the slide is placed in any other position, the sines or tangents of the angles denoted by any divisions on either of these special lines will vary in the same proportion as do the numbers which are opposite them on the $A$ line. In those rules in which lines of sines and tangents are given there is generally a scale of equal parts in which the length of the $\mathrm{D}$ line is divided into 500 or 1000 parts. If this is placed opposite the $\mathrm{D}$ line, with the ends of the two scales opposite one another, a table of logarithms will be seen; thus the logarithm of any number on the $\mathrm{D}$ line will be found opposite to it on the scale of equal parts.

Having pointed out the chief uses of a slide rule, it will be possible to describe the differences in construction in the several varieties. The most simple possible form is the original Gunter's scale to be found on any sector. With this and a pair of dividers calculations may be made, for if the dividors are set to the distance between any two numbers, any other pair of numbers which are found by the dividors to be the same distance apart will be in the same proportion, or have a common quotient, just as a common difference would be found if a scale of equal parts were used. This, however, is troublesome; but if the same principle is applied to a scale in the circular form the result is much more convenient. In this case angular distance takes the place of linear distance, and a pair of arms which can be opened to any angle can be moved round, and every pair of numbers covered will bear to one another a constant proportion depending on the extent of the angle. This is the principle of some of Dixon's rules shown at the Inventions Exhibition, near the arithmometers. In the well-known pocket instrument, the calculating circle of Boucher, an instrument like a watch, one hand is fixed and one is movable, and the face is also movable. There is another instrument of the same kind, in which the scale is drawn on a helical line. Here the scale and one hand are movable, and there is one fixed hand. This, which is Prof. Fuller's spiral rule, is made and exhibited by Stanley. Circular instruments are also made, in which scales slide over one another, which are in this respect like the straight rules. There is more advantage in the circular form than appears at first. In the straight rules the $\mathrm{A}$ and $\mathrm{B}$ lines are each double, the first and second halves are identical; this repetition of the scale is required in order that, however the slide may be placed, the part of each opposite to the other may contain at least a complete scale of numbers. In the circular form, however, the beginning and end of a single logarithmic scale meet, and so the scale itself is its own repetition both above and below. For this reason the openness of the divisors in a circular instrument is the same as in a straight rule, of which the length is six times, instead of three times, the diameter of the circular line.

Of the two types of instrument--one in which one slide works against another, generally straight, sometimes circular, and the other in which there is no slide but only a line divided logarithmically with a pair of hands, which type is always circular-which may be called respectively the slide and the index types, each has certain advantages. The slide form is preferable, in that each setting ot the slide furnishes a complete table of pairs of related numbers, as, for instance, of any English and foreign measure, of squares and roots on any scale, such as diameters and areas of circles, or of sines or tangents on any scale, so that, without moving the slide, any number of results may be read off, whereas with instruments of the index type the scale must be moved under the hands, or the hands over the scale, for each result. On the other hand, index instruments are more convenient than the usual slide rules in working out long expressions of the form $\frac{a \times b \times c \times d}{e \times f \times g}$, in which any of the terms may be squares, cubes, sines, or tangents, for the terms are taken alternately from the numerator and de- 
nominator and set in order with the fixed and movable hand until all are worked off, when the answer is found under the fixed hand. There is no necessity to observe any result till the process is complete; on the other hand, with slide instruments, each result of the form $\frac{a \times b}{e}$, $\frac{a \times b \times c}{e \times f}$, \&c., must be read and set before it can be operated upon by the next pair of factors. In Gravet's rules, however, this disadvantage of the straight form is removed by the addition of a cursor or sliding index, which in other ways is a great comfort.

All instruments of the index type suffer terribly from parallax, owing to the hands being above the face, so that they do not in practice give the accuracy that from the length of scale upon them might be expected.

This is especially the case in small instruments: for instance, Boucher's calculating circle, made in the form of a watch, is probably divided so accurately that on that score an error of one part in a thousand does not exist; yet, owing to parallax, the practical limit is about I-30o. This instrument has, besides the ordinary line, one on a double and one on a treble scale for squares and cubes, a line of sines, and another of equal parts for logarithms.

The possible accuracy of any instrument depends upon the length of the scale included between I and Io, called the radius, and also upon the linear accuracy with which a setting or reading can be made; this is at least twice as great in slide as in index instruments. In order to obtain great accuracy various means have been adopted whereby a great length of scale is brought within a small compass. Among slide instruments are Prof. Everett's "Universal Proportion Table," published by Longmans, Green, and Co., and General Hannyngton's slide rule, made and exhibited at the Inventions Exhibition by Aston and Mauder. In these the slide is made in the gridiron form. In Everett's instrument there are twenty bars, the total length of which is about I 3 feet; a scale of equal parts is also printed, so that logarithms can be read with it. In both of these instruments only simple proportions can be effected, unless special grids, divided on a double scale or trigonometrically, are provided. Far the most ingenious of all devices for obtaining a great length of radius in a comparatively short space is due to $\mathrm{Mr}$. Beauchamp Tower, whose name is well known in connection with the spherical engine. His instrument is a slide instrument consisting of two tapes running side by side over equal and independent rollers, but the tapes have a half twist in them, so that they have each only one surface and one edge. In this instrument, made privately for his own use, each tape is about $12 \frac{1}{2}$ feet long, and as both sides of the tape are used the radius is about 25 feet, and therefore, as far as openness of scale is concerned, it is equivalent to a straight rule 5o feet long, while the instrument itself is only just over 6 feet in length.

Slide rules of the index class can have a great length of scale more readily employed than others. Thus Prof. Fuller's helical instrument has its radius equal to $42 \frac{1}{2}$ feet, and is in openness of scale equivalent to a straight rule 85 feet long, while the box which contains it is only $17 \times 3 \frac{3}{4} \times 3 \frac{3}{4}$ inches inside measure. Dixon exhibits a special rule with the scale extending over Io concentric circles, but with this form a less degree of accuracy is attainable when using the inner than when using the outer circle. Thus the inner circle is equivalent to a straight rule 30 feet long and the outer to one 60 feet long. There is an outer circle equally and logarithmically divided to find logarithms. In another of Dixon's instruments, similar in size and form, there is the same outer circle for proportions and logarithms, and a series of inner circles divided so as to give sines, cosines, tangents, cotangents, secants, and cosecants. Each of these is on a board 14 inches square. Rules with very extended scales do not in practice give results with an accuracy which is proportional to their length, though the working accuracy is very much increased. They have this advantage, that they can be worked to their limit with ease, while with a well-divided pocket rule the errors of construction are beyond the limits of vision, and so the calculator is apt to strain his eyes to get results as accurate as possible. For instance, results obtained by a good pocket-rule one foot long can be trusted to a thousandth part; at the same rate Prof. Everett's should be accurate to a thirteen-thousandth part, and Prof. Fuller's to an eighty-five thousandth part. In practice a four and a ten-thousandth part are their limits. Again, instruments with very extended scales have only room for one line, so that simple proportions only and logarithms are all that can be directly obtained from them. For general use in the laboratory or elsewhere where calculations of every kind have to be made, the straight form, on the whole, seems most convenient, because of its portability, the quickness with which it can be worked, the diversity of operations that it will directly accomplish, and the extraordinary accuracy in comparison with other forms of the results to be obtained. Far the best instruments of this type that the writer has yet seen are those made by Tavernier-Gravet, of Paris, already alluded to. They are different to those generally used in England in that the line in the slide which works against the D line is itself a $\mathrm{D}$ line, so that squared proportions have to be performed by the aid of the cursor. This form has the further disadvantage that the inverted slide cannot be used for finding factors, which is a great loss; on the other hand, the two lower lines may be used for simple proportions, and they will give a double accuracy. On the whole, the original pattern with an A, B, C and D line seems preferable. Of the straight rules shown at the Inventions Exhibition those made by Stanley exceed all the others in workmanship and they are equal in this respect to the Gravet rule. Among them are rules for special purposes, as Hudson's scales and Ganga Ram's rules. Hudson's scales, which are made in card, each having two slides, are a marvel of constructive skill. Dixon shows his " triple radius double slide rule," with which very complex operations may be readily performed. Heath shows a slide rule for converting sidereal to mean solar time, or the reverse, correct to about 02 of a second, but this is not a slide rule proper, as the scales are not logarithmic.

There is entirely a different class of slide rule shown by Lieut. Thomson. In this there is, as usual, an A, B, and C line, but instead of the D line there is a " $\mathrm{P}$ " line, in which the distances, instead of being logarithmic, are logarithms of logarithms. By this instrument fractional powers may be found as readily as simple products or quotients. It has, however, this defect, that the scale converges so rapidly as the numbers ascend that high numbers can only be obtained with a proportionate accuracy far less than is possible with low numbers. It is one feature in the slide rule of ordinary construction that an error of reading of, say, I-Iooth of an inch will produce the same proportionate error in any part of the scale. This rule for involution is shown in the straight and circular form. It is right to mention that the same thing exactly was invented by the late Dr. Roget and published by him in the Phil. Trans. of 1815 .

No attempt has been made to give an account of every special form of rule that is made; those shown at the Exhibition and some other well-known forms, which well illustrate the different kinds of development, have been imperfectly described and the general principles on which all depend sufficiently explained to make evident the advantages of each type of instrument.

C. V. Boys 